\title{
Hybrid Liquid-Crystalline Block Copolymers II. X-Ray Diffraction Analysis of Polyester-Polymethacrylate Block Copolymers
}

\author{
Oriano Francescangeli, Michele Laus,* Maria Chiara Bignozzi,* Annino Sante Angeloni,* \\ Giancarlo GaLli, ${ }^{* *}$ and Emo ChIELlini**,† \\ Dipartimento di Scienze dei Materiali e della Terra, Università di Ancona, \\ Via Brecce Bianche, 60131 Ancona, Italy \\ * Dipartimento di Chimica Industriale e dei Materiali, Università di Bologna, \\ Viale Risorgimento 4, 40136 Bologna, Italy \\ ** Dipartimento di Chimica e Chimica Industriale, Università di Pisa, \\ Via Risorgimento 35, 56126 Pisa, Italy
}

(Received February 21, 1995)

\begin{abstract}
The molecular organization in the smectic mesophases of a series of main-chain/side-chain block copolymers was studied by X-ray diffraction on oriented specimens. The main-chain and the side-chain blocks were phase-separated and gave rise to two coexisting smectic domains with different layer periodicities. The orientation of the smectic planes in the distinct domains with respect to the fiber axis was very different in the various samples and depended on the relative length of the blocks. In the presence of relatively short side-chain blocks, the orientation of the main-chain block dominated and the smectic planes of the side-chain block were forced to be parallel to those of the main-chain block and perpendicular to the fiber axis. In contrast, when the side-chain block was sufficiently long it imposed its own orientation to the main-chain block. In this case, the smectic planes of the main-chain block were oriented parallel to those of the side-chain block and to the fiber axis.

KEY WORDS Block Copolymer / Liquid-Crystalline Copolymer/Polyester Block / Polymethacrylate Block / X-Ray Diffraction /
\end{abstract}

Main-chain/side-chain block copolymers constitute a novel and intriguing class of liquid-crystalline (LC) materials. ${ }^{1,2}$ Unusual morphologies and unconventional thermodynamic properties can be expected due to the intimate coupling between the main-chain and side-chain blocks which possess conflicting orientation characteristics of the polymer chain to the mesogenic groups. In general, the overall mesophase properties are foreseen to depend critically on the degree of compatibility between the two blocks. This in turn relies on several parameters such as the chemical nature of the blocks, the block length and block length distribution, the number and succession of blocks in a chain, and the overall composition. In addition to these chemical and molecular factors, each block may have specific tendency to pack in ordered structures of crystalline or mesomorphic type.

To evaluate the effects exerted by the different blocks on each other, we have prepared and studied a series of LC main-chain/side-chain block copolymers 1a-d constituted by a side-chain LC polymethacrylate block and a main-chain LC polyester block ${ }^{2}$ of various lengths (Figure 1).

Thermal and dynamic-mechanical analyses of block copolymers 1a-d had shown that the two chemically different blocks were partly phase-separated in the glassy and LC phases and underwent distinct transitions, which could also be monitored by X-ray diffraction measurements. $^{2}$ The presence of smectic $\mathrm{C}$ and nematic mesophases for the polyester block and of smectic A and nematic mesophases for the polymethacrylate block was evidenced. However, significant deviations of the phase transition parameters of both blocks from those expected from the structurally analogous homopolymers were

\footnotetext{
† To whom all correspondence should be addressed.
}

observed. In particular, the stability and degree of order of the LC phases relevant to both the main-chain and side-chain blocks were lower than the ones of the corresponding homopolymers. These data indicate the existence of a certain degree of miscibility between the main-chain and side-chain blocks thus possibly producing an interphasic region.

With the aim of providing a detailed description of the molecular organization in the smectic mesophases, in the present paper we report an X-ray diffraction study on oriented fibers of main-chain/side-chain block copolymers $1 \mathbf{a}-\mathbf{d}$.

\section{EXPERIMENTAL}

\section{Materials}

Block copolymers were synthesized via two successive polycondensation and free-radical polymerization processes. ${ }^{2}$ Four samples 1a-d were prepared with different compositions. The content of the polymethacrylate block, as evaluated by ${ }^{1} \mathrm{H}$ NMR, ranged from 35 to $79 \mathrm{wt} \%$. By considering that in all the copolymer samples the polyester block had $M_{n}=3400,{ }^{2} M_{n}$ values in the range 5500 to 16500 were evaluated for the $\mathrm{AB}$ copolymers by ${ }^{1} \mathrm{H}$ NMR (Table I). $M_{n}$ values in the range 18000 to 33000 and $M_{w} / M_{n}$ comprised between

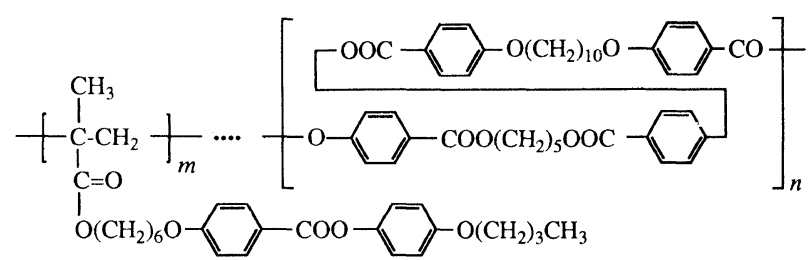

Figure 1. Simplified structure of block copolymers $1 \mathbf{a}-\mathbf{d}$. 
Table I. Composition data and molar mass characteristics of block copolymers $\mathbf{1 a - d}$ and their respective polymethacrylate block

\begin{tabular}{|c|c|c|c|c|}
\hline \multirow{2}{*}{ Sample } & \multirow{2}{*}{$M_{n}^{\mathrm{a}}$} & \multirow{2}{*}{$M_{w} / M_{n}^{\mathrm{a}}$} & \multicolumn{2}{|c|}{ Polymethacrylate block } \\
\hline & & & wt $\%$ b & $M_{n}^{\mathrm{a}}$ \\
\hline $1 \mathbf{a}$ & $18000(5500)$ & 1.7 & 35 & $14000(2000)$ \\
\hline lb & $21000(8500)$ & 1.8 & 59 & $18000(5000)$ \\
\hline $1 \mathrm{c}$ & $24000(10500)$ & 2.0 & 67 & $21000(7000)$ \\
\hline 1d & $33000(16500)$ & 3.4 & 79 & $30000(13000)$ \\
\hline
\end{tabular}

${ }^{a}$ By SEC, with polystyrene calibration (values in parentheses were

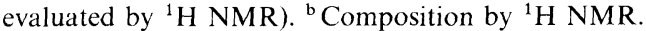

1.7 and 3.4 were evaluated by SEC using the polystyrene calibration curve (Table I).

\section{Characterization}

Fibers were obtained by pulling up with tweezers the highly viscous smectic melt at temperatures between 350 and $365 \mathrm{~K}$. X-Ray diffraction photographs on oriented samples were obtained with a Rigaku-Denki RU300 rotating anode generator equipped with a pinhole flat camera. Ni-filtered $\mathrm{Cu}-K_{\alpha}$ radiation $(\lambda=1.54 \AA)$ was used. The distance between sample and film was $75 \mathrm{~mm}$. Depending on the intensity of the diffraction signals, different exposure times of the films were required, varying between seven and nine hours, with voltage $40 \mathrm{kV}$ and current $200 \mathrm{~mA}$. The optical density of the X-ray diffraction photographs was determined by digitalizing the X-ray patterns, using the Kodak photo-CD system. The values of the integrated intensity of the different reflections were evaluated after background subtraction by numerical integration of the digitalized signal using the NIH image processing (version 1.55, public domain; FTP address: zippy.nimh.nih.gov) and analysis program. Colour images were obtained by elaborating the digitalized signal by Transform (version 2.1, Spyglass, Inc., 701 Devonshire Dr., C-17, Champaign, IL 61820) image processing program.

\section{RESULTS AND DISCUSSION}

The preparation and thermal behavior of block copolymers 1a-d were described in some detail in a previous paper. ${ }^{2}$ The content of the polymethacrylate block was comprised between 35 and $79 \mathrm{wt} \%$. Due to the synthetic procedure that leads mainly to AB diblock copolymers, ${ }^{2} M_{n}$ was 3400 for the polyester block in each block copolymer, as determined by SEC using the universal calibration method with suitable polyester standards. Thus, $M_{n}$ values in the range 5500 to 16500 were estimated by ${ }^{1} \mathrm{H}$ NMR (Table I). The $M_{n}$ values evaluated by SEC using polystyrene calibration varied from 18000 (1a) to 33000 (1d), and therefore $M_{n}$ of the polymethacrylate block varied from $\approx 14000$ (1a) to $\approx 30000$ (1d) (Table I). Polyester 2, structurally analogous to the polyester block, was amorphous and formed smectic $\mathrm{C}$ and nematic mesophases. ${ }^{2,3}$ Polymethacrylate 3, structurally analogous to the polymethacrylate block, was also amorphous and exhibited smectic A and nematic mesophases. ${ }^{2,4}$ Copolymers $1 \mathbf{a}-\mathbf{d}$ exhibited all the above phases in a sequence with increasing temperature, the mesophases of the polyester block being stable at higher temperatures. In particular, two smectic phases coexisted over well defined ranges of temperature which depended on the copolymer composition. The occurrence of individual transitions associated with both the main-chain and side-chain blocks indicated that the two blocks were separated in the LC phases.

To understand the molecular organization in the smectic mesophases of the block copolymers, an X-ray study was performed on oriented mesophases produced by drawing fibers at temperatures between 350 and $365 \mathrm{~K}$ in the region where the smectic mesophase of the polymethacrylate block and the smectic mesophase of the polyester block coexisted, and cooling them in air to room temperature. The X-ray diffraction diagrams of the oriented mesophases of the four copolymers are shown in Figure 2. To better visualize the X-ray patterns, their intensity contour maps in the small-angle region are reported in Figure 3. The anisotropy of the patterns clearly shows that a high degree of molecular orientation can be achieved by drawing fibers from the mesophase. Two sharp reflections were clearly visible in the smallangle region of all the spectra, which corresponded to periodicities $\left(d_{1}\right.$ and $\left.d_{2}\right)$ of 20.0 and $29.0 \AA$, respectively. The former periodicity was equal to the layer spacing of the smectic $\mathrm{C}$ mesophase of polyester $\mathbf{2}$, and the latter to that of the smectic A mesophase of polymethacrylate 3 . Therefore, the phase-separated polyester and polymethacrylate blocks gave rise to two coexisting smectic phases. In general, in the wide-angle region a rather diffuse halo was detected due to the liquid-like arrangement of the mesogens within the smectic (A or C) layers (intermolecular distance $D \approx$ $4.6 \AA$ ). However, because of the overlapping signals of the two different blocks in this angular region, it was not possible to identify the exact nature of the smectic mesophases. The relative orientations of the smectic planes in the distinct domains with respect to the fiber asix were very different in samples with different compositions. For sample 1a (Figures 2A and 3A), the small-angle reflection at $d_{2}=29.0 \AA$ appeared as a weak meridional signal, whereas the contribution of the reflection at $d_{1}=20.0 \AA$ was a ring of not uniform intensity distribution. Well defined maxima were seen on the meridian but lower intensity signals were also recorded on the equator. The wide-angle signals consisted of two diffuse crescents centered on the equator. For sample 1d (Figures 2D and 3D), the small-angle reflection at $d_{2}=29.0 \AA$ was localized on the equator. Well defined intensity maxima in the signal at $d_{1}=20.0 \AA$ were observed on the equator, whereas low intensity signals were also present on the meridian. The wide-angle pattern comprised two wide diffuse crescents centered around the meridional line. An intermediate behavior was found for samples $\mathbf{1 b}$ and $\mathbf{1 c}$. In the diffraction spectrum of $\mathbf{1 b}$ (Figures 2B and 3B), the intensity of the reflection at $20.0 \AA$ was principally distributed along the meridian. The reflection corresponding to $29.0 \AA$ appeared as a sharp signal localized on the equator. The wide-angle diffuse diffractions exhibited no preferential orientation. A smaller degree of orientation was observed in the X-ray spectrum of sample 1c (Figures $2 \mathrm{C}$ and $3 \mathrm{C}$ ) compared to the previous samples. The small-angle signal at $d_{2}=$ 


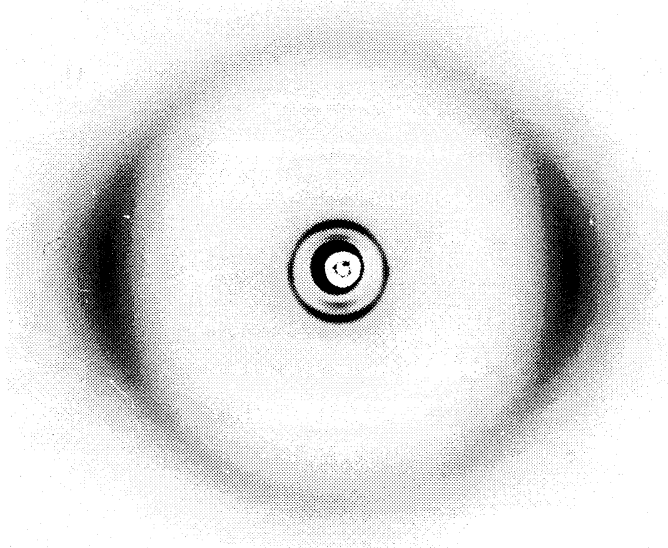

(A)

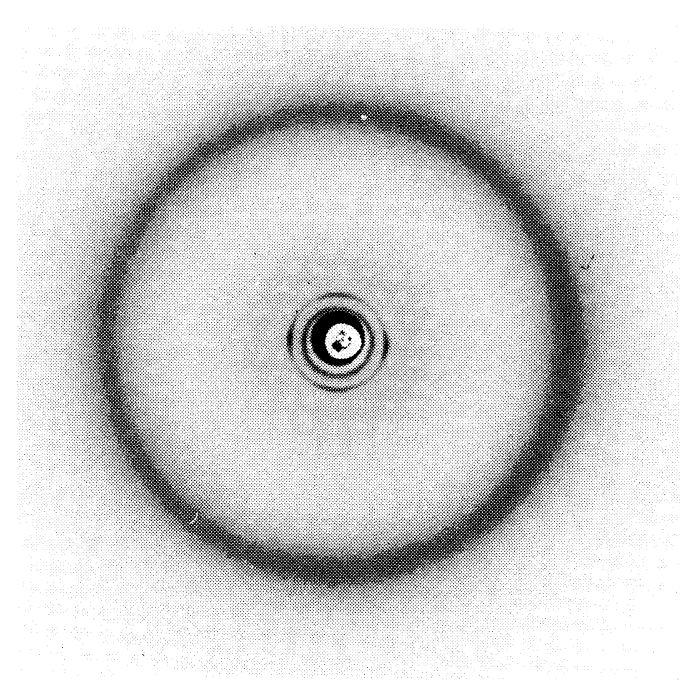

(C)

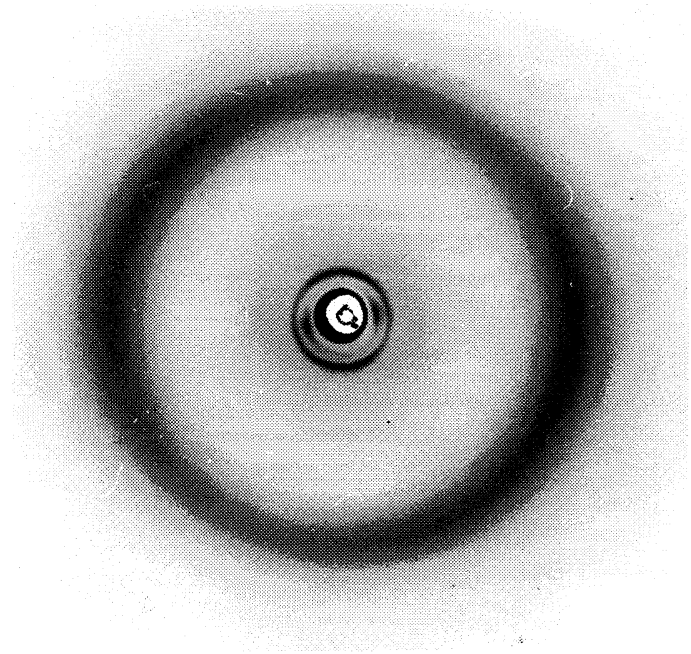

(B)

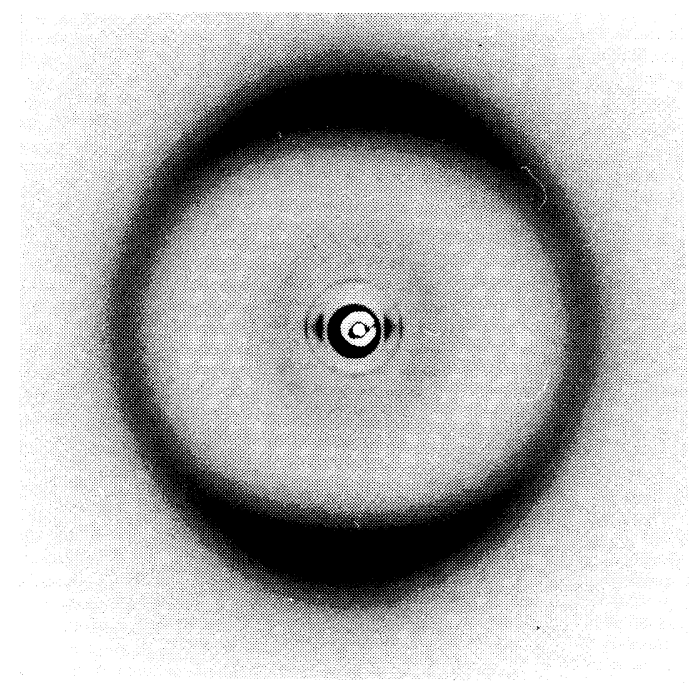

(D)

Figure 2. Fiber X-ray diffraction pattern (vertical fiber axis) of block copolymers 1a (A), 1b (B), 1c (C), and 1d (D).

$29.0 \AA$ was a ring of not uniform intensity distribution with diffuse intensity maxima on the equator, whereas four well defined maxima on the equatorial and meridional lines, were detected in the small-angle signal corresponding to $d_{1}=20.0 \AA$.

The peculiar orientations of block copolymers $1 \mathbf{a}-\mathbf{d}$ result from the concomitant tendencies of the polymer chain of the polyester block to orient normal to the smectic planes and of the polymer chain of the sidechain block to orient parallel to the smectic planes. Due to these opposite orientation characteristics of the different blocks, three limiting local structural arrangements should be considered (Figure 4). In structure I, the polymer backbones of both the main-chain and side-chain blocks are aligned along the fiber axis, and accordingly the smectic planes of the main-chain block domain are perpendicular to the smectic planes of the side-chain block domain. In structure II, the polymer backbone of the main-chain block is aligned along the fiber axis, whereas the polymer backbone of the sidechain block is aligned perpendicular to the fiber axis. Therefore, the smectic planes of both the main-chain and side-chain block domains are parallel to each other and perpendicular to the fiber axis. The opposite situation can be seen in structure III, where the polymer backbone of the side-chain block is aligned along the fiber axis, whereas the polymer backbone of the main-chain block is aligned perpendicular to the fiber axis. Accordingly, the smectic planes of both the main-chain and side-chain block domains are parallel to the fiber axis and to each other. While structures II and III are equivalent in unoriented, powder samples, they can be differentiated in oriented, fiber specimens. The X-ray diffraction spectrum of sample 1a, in which the polymethacrylate block is the shortest one, is very similar to the one expected considering the chain arrangement in structure II. In contrast, the spectra of samples $\mathbf{1 b}$, 1c, and $\mathbf{1 d}$ can be obtained by combining to different extents those relevant to structures I and III. From the intensity contour maps of the small-angle X-ray spectra we can estimate the relative amount of the two different structural arrangements of the smectic layers. To this purpose, it is sufficient to observe that only structure I contributes to the meridional reflection at $d_{1}=20.0 \AA$, whereas only structure 


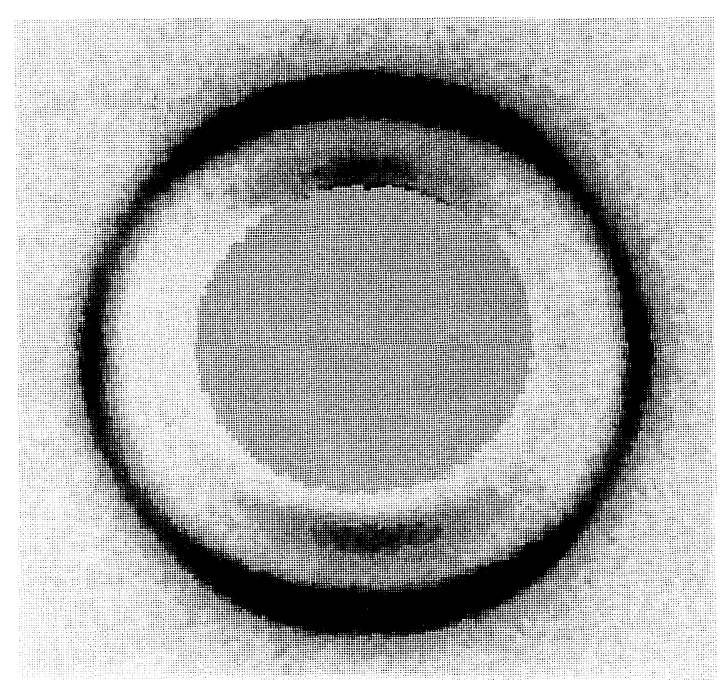

(A)

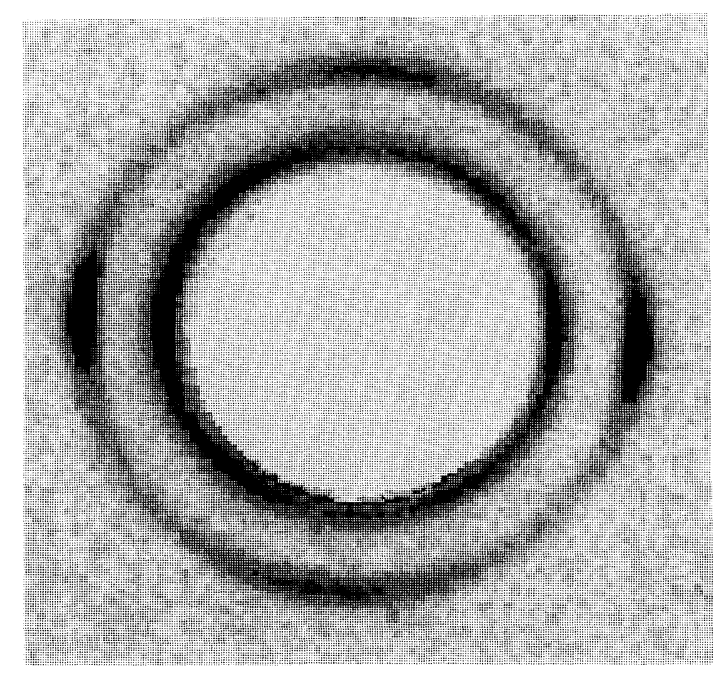

(C)

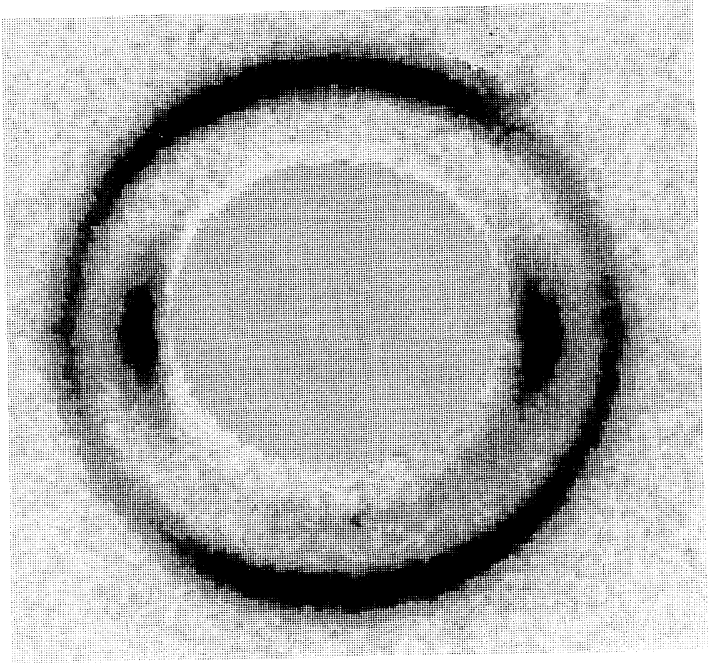

(B)

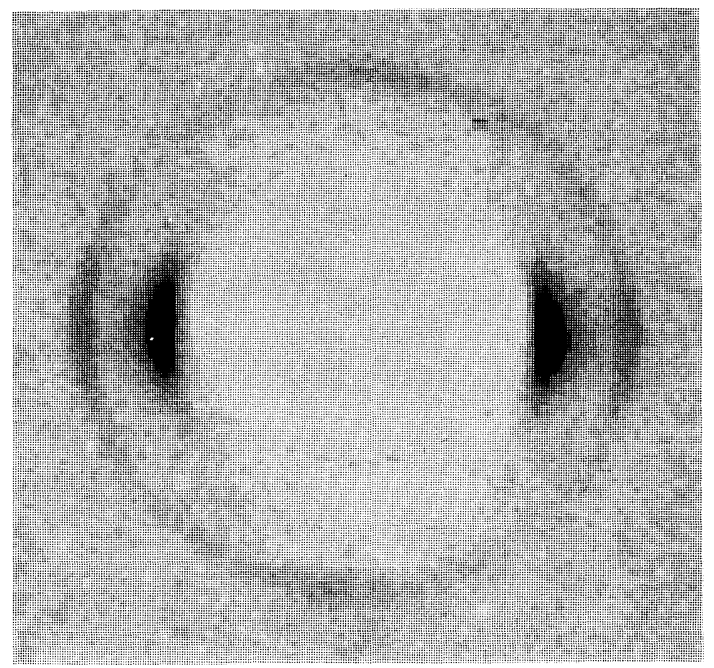

(D)

Figure 3. Intensity contour map of the small-angle diffraction region of block copolymers $\mathbf{1 a}(\mathrm{A}), \mathbf{1 b}(\mathrm{B}), \mathbf{1 c}(\mathrm{C})$, and $\mathbf{1 d}$ (D) (vertical fiber axis).

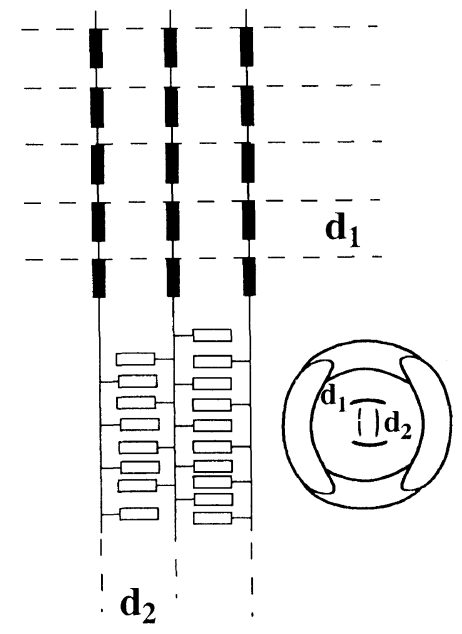

(A)

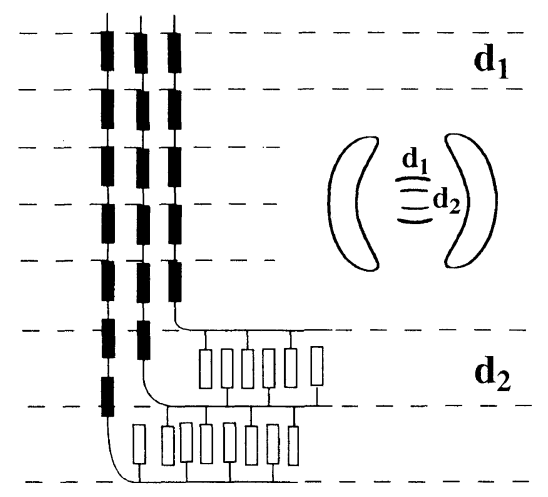

(B)

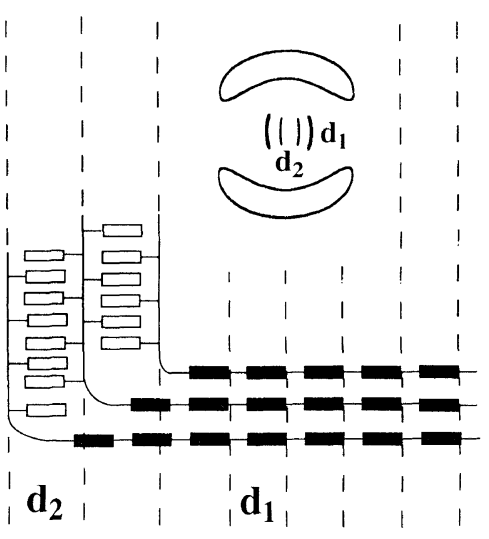

(C)

Figure 4. Model structures I (A), II (B), and III (C) of polymer chain arrangements of block copolymers 1a - d with a schematic representation of their expected small-angle X-ray diffraction diagram (possible interfacial regions are not considered).

III contributes to the equatorial reflection at the same $d_{1}$ value. Accordingly, the ratio of the intensity of the meridional diffraction $\left(I_{\mathrm{me}}\right)$ over the intensity of the total diffraction $\left(I_{\mathrm{me}}+I_{\mathrm{eq}}\right)$ for the small-angle signal of the polyester block provides an approximate evaluation of the percent contribution from structure I (Table II). On 
Table II. Diffraction intensity ratios for the small-angle region $^{\mathrm{a}}$ of the polyester block of block copolymers $\mathbf{1 b}$ - d

\begin{tabular}{ccc}
\hline Sample & $I_{\mathrm{me}} / I_{\mathrm{eq}}$ & $\begin{array}{c}I_{\mathrm{me}} /\left(I_{\mathrm{eq}}+I_{\mathrm{me}}\right) \\
(\%)\end{array}$ \\
\hline 1b & $5.1 \pm 0.3$ & $84 \pm 1$ \\
1c & $0.65 \pm 0.04$ & $39 \pm 3$ \\
1d & $0.35 \pm 0.04$ & $26 \pm 4$
\end{tabular}

${ }^{\text {a }}$ Diffraction intensity of the reflection at $d_{1}=20.0 \AA$ on the meridian $\left(I_{\mathrm{me}}\right)$ and the equator $\left(I_{\mathrm{eq}}\right)$.

passing from block copolymer $\mathbf{1 b}$ to $\mathbf{1 d}$, the contribution of structure I decreases, whereas the one of structure III increases.

The orientation behavior of block copolymer $1 \mathbf{a}-\mathbf{d}$ under a mechanical field and the mutual influence of the chemically different blocks was correlated to the block lengths. As the main-chain block length was constant in all copolymers the distinct orientation propensities of the smectic domains with respect to the fiber axis essentially depended on the length of the polymethacrylate block. When the polymethacrylate block was relatively short like in $\mathbf{1 a}$, the orientation of the polyester block dominated and the smectic planes of the side-chain block were forced to pack parallel to those of the main-chain block and perpendicular to the fiber axis (structure II). On the contrary, when the polymethacrylate block was sufficiently long like in 1d, it imposed its own orientation characteristics on the polyester block and the smectic planes of the main-chain block aligned parallel to those of the side-chain block and to the fiber axis (structure III). For intermediate chain lengths, the orientation characteristics of both the different blocks played a role thus forcing the smectic planes of the side-chain and main-chain blocks to assemble perpendicular to each other and perpendicular and parallel to the fiber axis, respectively (structure I).

\section{CONCLUSIONS}

The molecular organization in the smectic mesophases of main-chain/side-chain block copolymers 1a-d based on liquid-crystalline polyester and polymethacrylate blocks was studied by X-ray diffraction on oriented fibers. The main-chain and side-chain blocks were phaseseparated and gave rise to the coexisting smectic phases with different layer periodicities. The relative orientations of the chains and the smectic planes differed in the various samples and were correlated to the length of the main-chain and side-chain blocks. For short side-chain blocks, the smectic planes of the side-chain block were forced to orient parallel to those of the main-chain. In contrast, when the side-chain block was sufficiently long it imposed its own orientation characteristics on the main-chain block. In this case, the smectic planes of the main-chain block were parallel to those of the sidechain block. For intermediate chain lengths, both the chemically different blocks imposed their own orientation characteristics. Accordingly, the smectic planes of the side-chain and main-chain blocks were perpendicular to each other and perpendicular and parallel respectively to the fiber axis. As a final remark, it should be stressed that the block length polydispersity, inherent in the present block copolymer system, does not allow a precise evaluation of a critical chain length corresponding to which the transition between the orientational regimes can occur. In addition, the continuous or discontinuous nature of this transition with respect to the block chain length is still to be clarified with other pertinent experiments using samples with different molar mass and narrow molar mass distribution.

Acknowledgment. This work was supported from the Ministero dell'Università e della Ricerca Scientifica e Tecnologica of Italy.

\section{REFERENCES}

1. G. Galli, E. Chiellini, M. Laus, M. C. Bignozzi, A. S. Angeloni, and O. Francescangeli, Makromol Chem., 195, 2247 (1994).

2. M. Laus, M. C. Bignozzi, A. S. Angeloni, O. Francescangeli, G. Galli, and E. Chiellini, Polym. J., 27, 993 (1995).

3. O. Francescangeli, B. Yang, G. Albertini, A. S. Angeloni, M. Laus, E. Chiellini, and G. Galli, Liq. Cryst., 13, 353 (1993).

4. R. Zentel and H. Ringsdorf, Makromol. Chem., Rapid Commun., 5, 393 (1984). 